\title{
Equivalence of conventional and fast late gadolinium enhancement (LGE) techniques for quantitative evaluation of fibrosis in ischemic and non-ischemic cardiac disease - Save the Time!
}

\author{
Fabian Muehlberg ${ }^{*}$, Kristin Arnhold, Stephanie Funk, Marcel Prothmann, Andre Rudolph, \\ Florian von Knobelsdorff-Brenkenhoff, Jeanette Schulz-Menger
}

From 19th Annual SCMR Scientific Sessions

Los Angeles, CA, USA. 27-30 January 2016

\section{Background}

Segmented single-slice/single-breath-hold 2D phase-sensitive inversion recovery (2D-PSIR) sequences are the gold standard for evaluation of myocardial fibrosis. Aim of this study was to assess the accuracy of novel free-breathing or single-breath-hold LGE sequences to detect and quantify myocardial fibrosis in patients with different entities.

\section{Methods}

Patients with myocardial infarction $(\mathrm{n}=45)$, myocarditis $(\mathrm{n}=25)$ or hypertrophic cardiomyopathy $(\mathrm{HCM})(\mathrm{n}=$ 15) were prospectively enrolled. After administration of gadolinium contrast agent, LGE images were acquired ECG-gated in short axis slices (slice thickness $7 \mathrm{~mm}$, no gap) using 4 different LGE sequences: (1) conventional segmented $2 \mathrm{D}$ phase-sensitive inversion recovery in single-slice/single-breath-hold technique (2D-PSIR; gold standard; TR $744 \mathrm{~ms}$, TE 5,17 ms, voxel size $1.4 \times 1.4 \times$ $7.0 \mathrm{~mm}$ ), (2) single-breath-hold 3D-IR sequence (3D-IR bh; TR $924 \mathrm{~ms}$, TE $1.06 \mathrm{~ms}$, voxel size $1.9 \times 1.9 \times 7.0$ $\mathrm{mm}$ ), (3) single breath-hold 3D-SSFP sequence (3DSSFP; TE $700 \mathrm{~ms}$, TE $1.05 \mathrm{~ms}$, voxel size $1.9 \times 1.9 \times 7.0$ $\mathrm{mm}$ ) and (4) non-breath-hold technique (3D-IR nbh). (Figure 1) For all techniques, inversion time was individually adjusted to null the remote myocardium. Myocardial fibrosis was quantitatively assessed using a semiautomated threshold method; positive LGE was defined as signal intensity 6 standard deviations (SD) above signal intensity of remote myocardium for myocardial

\footnotetext{
Working Group on Cardiovascular MRI, Charité University Medicine and
} HELIOS Clinics, Berlin, Germany

\section{Conclusions}

Fast 3D-SSFP, 3D-IR and conventional segmented 2DPSIR sequences are equivalent techniques for the assessment of myocardial fibrosis, independent of an ischemic or non-ischemic etiology. Due to the minimized acquisition time they shorten scan protocols by up to 6 minutes. 


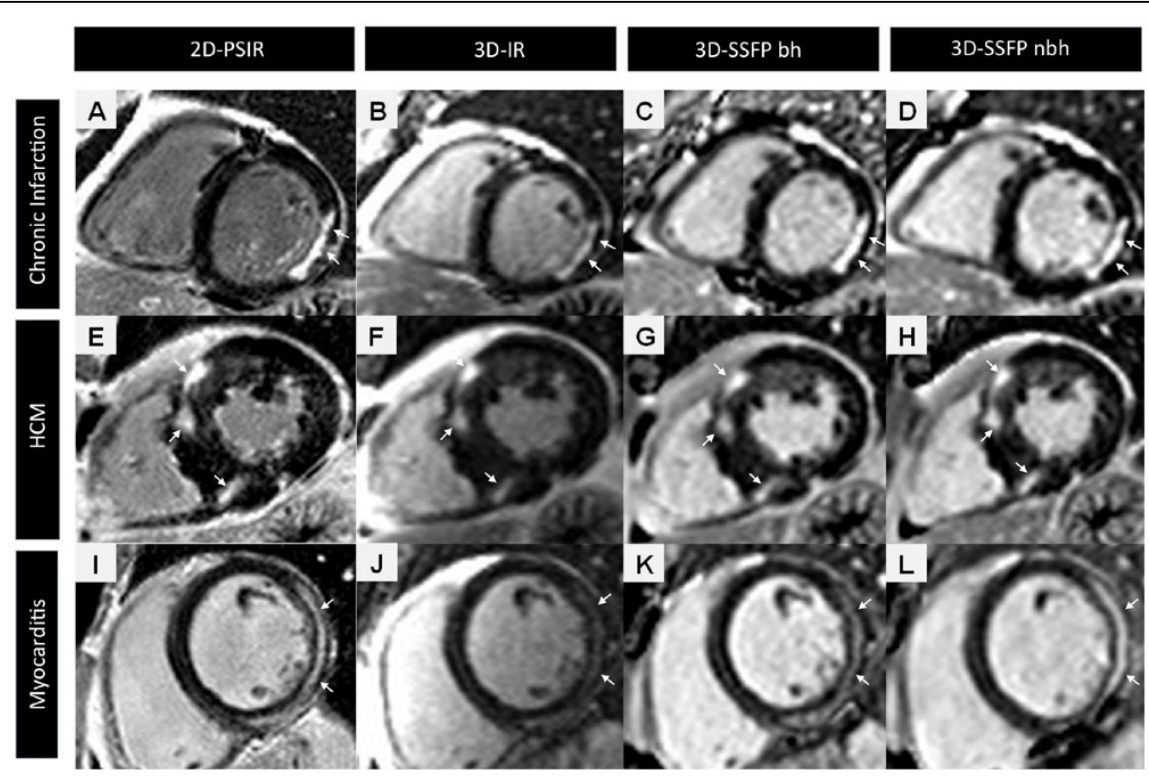

Figure 1 LGE images of three patients with chronic ischemis infarction (A-D), HCM (E-H) and acute myocarditis (I-L). Arrows indicate typical LGE localization. Horizontal rows display corresponding slices of LGE in the same patient using conventional segmented $2 \mathrm{~d}$-PSIR (A;E;), 3D-IR (B;F;J), 3D-SSFP bh (C; G;K) and 3D-SSFP nbh 9D;H;L).

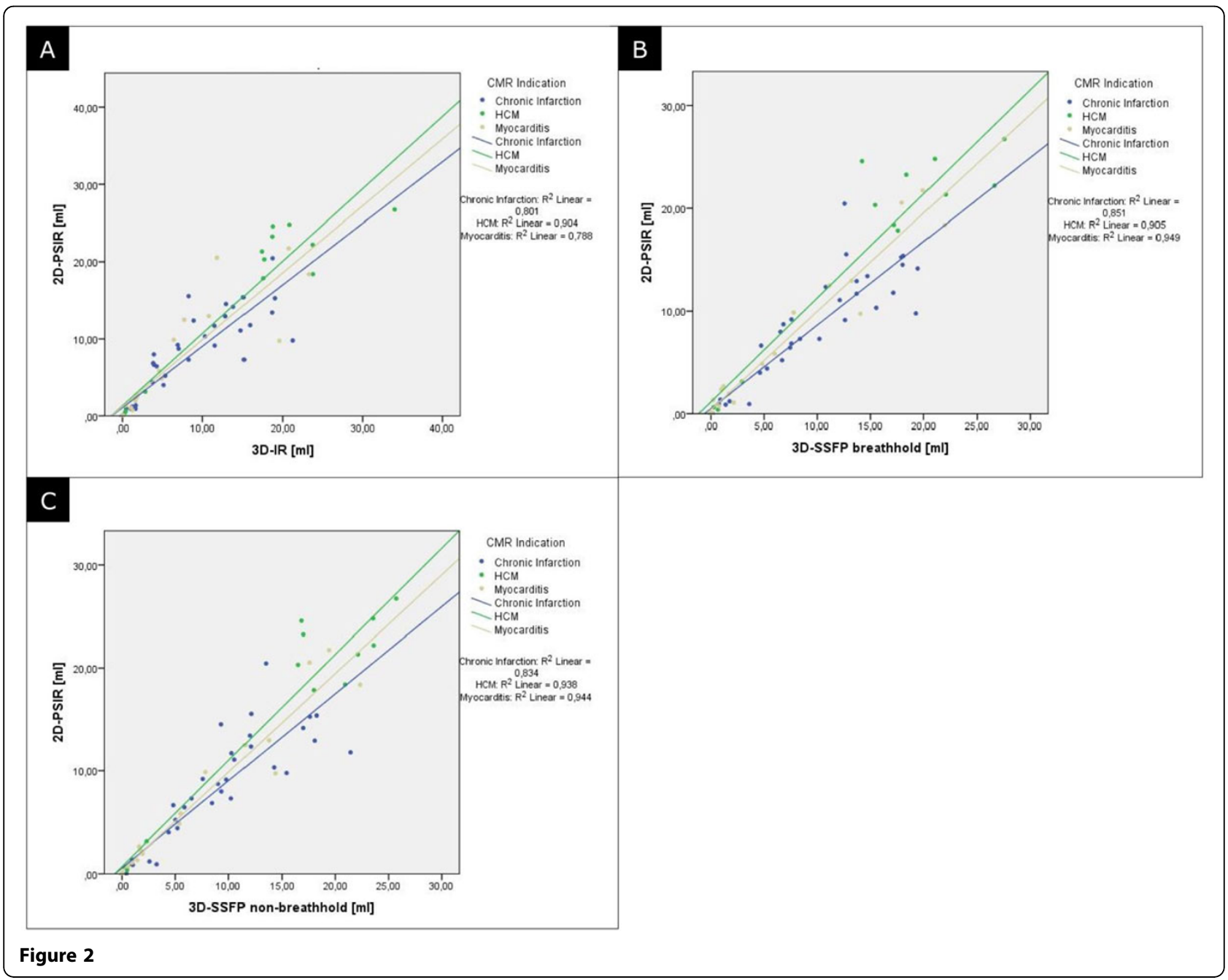


doi:10.1186/1532-429X-18-S1-Q64

Cite this article as: Muehlberg et al: Equivalence of conventional and fast late gadolinium enhancement (LGE) techniques for quantitative evaluation of fibrosis in ischemic and non-ischemic cardiac disease Save the Time!. Journal of Cardiovascular Magnetic Resonance 201618

(Suppl 1):Q64.

Submit your next manuscript to BioMed Central and take full advantage of:

- Convenient online submission

- Thorough peer review

- No space constraints or color figure charges

- Immediate publication on acceptance

- Inclusion in PubMed, CAS, Scopus and Google Scholar

- Research which is freely available for redistribution

Submit your manuscript at www.biomedcentral.com/submit

() Biomed Central 